\title{
Vascular endothelial growth factor receptor tyrosine kinase inhibitors versus bevacizumab in metastatic colorectal cancer: A systematic review and meta-analysis
}

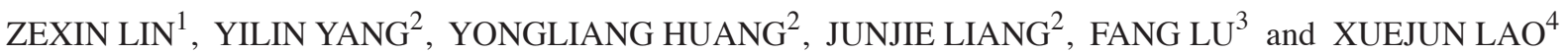 \\ ${ }^{1}$ Department of General Surgery, The First Clinical Medicine School of Jinan University, \\ Affiliated Shantou Hospital of Sun Yat-Sen University; Departments of ${ }^{2}$ General Surgery and ${ }^{3}$ Orthopedics, \\ The First Clinical Medicine School of Jinan University; ${ }^{4}$ Department of General Surgery, \\ The First Affiliated Hospital of Jinan University, Guangzhou, Guangdong, P.R. China
}

Received January 29, 2015; Accepted April 24, 2015

DOI: $10.3892 / \mathrm{mco} .2015 .572$

\begin{abstract}
Bevacizumab has demonstrated a survival benefit in patients with metastatic colorectal cancer (mCRC) when combined with chemotherapy. Several randomized clinical trials comparing the efficacy and toxicity of vascular endothelial growth factor receptor (VEGFR) tyrosine kinase inhibitors (TKIs) against bevacizumab have been reported. The present meta-analysis was conducted to identify the potentially significant benefit of the combined treatment regimens in patients with mCRC. PubMed, Embase and Cochrane Library databases were searched for the randomized controlled trials published on or before September 2014, which compared the efficacy and toxicity of VEGFR TKIs with bevacizumab in combination with chemotherapy in patients with mCRC. The primary endpoints included progression-free survival (PFS), overall survival (OS) and overall response rate (ORR), and secondary endpoints were the toxicity profiles. Relative risks (RRs) with 95\% confidence intervals (CIs) for response rate and adverse events (AEs) were calculated, as well as hazard ratios (HRs) for PFS and OS. The final analysis included 4 studies comprising a total of 1,929 intent-to-treat patients with mCRC, which compared VEGFR TKIs (cediranib and axitinib) plus chemotherapy with bevacizumab plus chemotherapy. Results demonstrated that VEGFR TKIs plus chemotherapy significantly resulted in a modest but significantly shorter PFS [hazard ratio (HR), 1.12; 95\% CI, 1.00-1.25; $\mathrm{P}=0.05$ ] compared with that of bevacizumab plus chemotherapy but not in OS (HR, 1.10; 95\% CI, 0.88-1.17; P=0.87) and ORR (RR, 0.95;
\end{abstract}

Correspondence to: Professor Xuejun Lao, Department of General Surgery, The First Affiliated Hospital of Jinan University, 613 West Huangpu Road, Guangzhou, Guangdong 510632, P.R. China E-mail: foxlxj2k@126.com

Key words: advanced or metastatic colorectal cancer, bevacizumab, vascular endothelial growth factor receptor tyrosine kinase inhibitor, chemotherapy, meta-analysis
95\% CI, 0.85-1.05; $\mathrm{P}=0.30$ ). VEGFR TKIs treatment showed a less favorable AE profile compared with bevacizumab, with higher rates of grade-III/IV diarrhea, fatigue, hypertension, neutropenia and thrombocytopenia, whereas a higher incidence of peripheral neuropathy associated with the bevacizumab group was observed. In conclusion, the addition of VEGFR TKIs to chemotherapy resulted in a modest but significantly shorter PFS but not in OS and ORR compared with bevacizumab. The VEGFR TKIs group showed a less favorable AE profile with higher rates of diarrhea, fatigue, hypertension, neutropenia and thrombocytopenia, whereas a higher incidence of peripheral neuropathy associated with the bevacizumab was observed.

\section{Introduction}

Colorectal cancer (CRC) is the third most common cause of malignancy in men and women in the United States. The prognosis is poor for patients with metastatic colorectal cancer (mCRC) and the 5-year survival rate for them is $\sim 12 \%$ (1). Standard first-line chemotherapy regimens for mCRC include 5-fluorouracil (5-FU)/leucovorin/oxaliplatin (FOLFOX) and 5-FU/leucovorin/irinotecan (FOLFIRI) (2). These two have incrementally led to improved overall response rates (ORR), progression-free survival (PFS) and overall survival (OS) in first-line regimens. However, the GERCOR study, which evaluated the efficacies of FOLFOX and FOLFIRI as first- and second-line therapies in patients with mCRC, demonstrated that the clinical benefit was greatly reduced with second-line treatment (3). More effective options are required to further improve outcomes.

As a key factor of tumor growth and metastasis, the vascular endothelial growth factor (VEGF) regulates normal and pathological angiogenesis, and activates multiple signaling networks that promote endothelial cell growth, migration and vascular permeability (4). A clinically validated therapeutic strategy to target the VEGF signaling axis in patients has been demonstrated with advanced mCRC. The VEGF monoclonal antibody bevacizumab (Avastin ${ }^{\circledR}$; Genentech, San Francisco, CA, USA), has demonstrated a clinical benefit in patients with 
mCRC when combined with chemotherapy in a randomized, phase III study, in which the addition of bevacizumab to oxaliplatin, fluorouracil and leucovorin (FOLFOX4) significantly prolonged PFS [7.3 vs. 4.7 months; hazard ratio (HR), 0.61; $\mathrm{P}<0.0001]$ and $\mathrm{OS}(12.9$ vs. 10.8 months; HR, 0.75; $\mathrm{P}=0.0011)$ compared with FOLFOX4 alone (5).

VEGF receptors (VEGFR) tyrosine kinase inhibitors (TKIs), such as cediranib and axitinib, have shown antitumor activity in patients with mCRC. Cediranib is an oral, highly potent VEGF TKI with activity against all three VEGFRs $(6,7)$. A randomized, phase III study (HORIZON II) of cediranib + FOLFOX/CAPOX versus placebo + FOLFOX/CAPOX for mCRC demonstrated that the addition of cediranib to chemotherapy prolonged PFS, but did not significantly improve OS (8). Axitinib, a potent and selective second-generation inhibitor of VEGFRs 1-3 (9), has shown promising single-agent activity against a variety of tumor types, including metastatic renal cell carcinoma, melanoma, thyroid cancer and non-small-cell lung cancer (10-14). As opposed to bevacizumab, it specifically binds VEGF-A, and cediranib and axitinib act directly at VEGFR 1-3 and may result in a more complete blockade of VEGF signaling. Several RTCs have been conducted to investigate efficacy and toxicity of VEGFR TKIs versus bevacizumab in combination with chemotherapy in patients with mCRC. However, the conclusions are not consistent. Therefore, the present meta-analysis was performed to evaluate the randomized controlled trials (RCTs) and compare the efficacy and toxicity of VEGFR TKIs plus chemotherapy with bevacizumab plus chemotherapy in patients with mCRC.

\section{Materials and methods}

Search criteria. PubMed, Embase and the Central Registry of Controlled Trials of the Cochrane Library were searched for all the relevant trials on or before September 2014, which compared efficacy and toxicity of VEGFR TKIs with bevacizumab in combination with chemotherapy in patients with mCRC. The following keywords were used: 'Advanced colorectal cancer' OR 'metastatic colorectal cancer' AND 'randomized controlled trial' AND 'bevacizumab' AND 'VEGFR TKIs' OR 'cediranib' OR 'axitinib' OR 'sunitinib'. Abstracts presented at the annual meeting of the American Society of Clinical Oncology (ASCO) and the European Society for Medical Oncology (ESMO) were also searched, and the reference lists of all the identified relevant studies for this topic were manually examined.

The inclusion criteria were as follows: i) Patients with histologically confirmed mCRC; ii) RCTs; iii) experimental and control groups treated by VEGFR TKI and bevacizumab respectively, and experimental group treated by VEGFR TKI plus the chemotherapy, while control group received bevacizumab plus the chemotherapy, and not confounded by additional biological agents or interventions; iv) trials should be explicit regarding numbers of cases in experimental and control groups, as well as the cases that finished the trials; and v) clinical index included PFS, OS, ORR and adverse events (AEs).

The exclusion criteria were: i) Trials that included patients with major comorbidities or second tumors were excluded; ii) quasi-randomized studies that were considered to possess insufficient quality; and iii) trials included adjuvant chemotherapy within 6 months or concomitant interventions were excluded.

Quality assessment. Quality of study methodology was scored using the methods reported by Jadad et al (15) and Kjaergard et al (16). This is a five-point scale, with one point awarded for each quality criterion (17).

Data extraction and statistical analysis. Two investigators (Y.L Huang and F. Lu) independently extracted the data from all the included studies according to the inclusion criteria listed. Disagreements were resolved by discussion with an independent expert (Y.L. Yang). The following information was sought: First author, year of publication, number of patients, number of patients eligible for response, gender rate, mean age, ORR, median OS and PFS, and data on AEs/toxicities, such as hypertension, vomiting, diarrhea, fatigue and neutropenia, thrombocytopenia and peripheral neuropathy.

Meta-analysis was carried out by RevMan 5.0 provided by the Cochrane Collaboration (Oxford, UK). HR for PFS and OS, relative risks (RR) for ORR and AEs with 95\% confidence intervals (CI) were calculated. To test the statistical heterogeneity for each trial, a Cochrane's $Q$ test was performed, and if $\mathrm{P}<0.1$, the assumption of homogeneity was considered invalid and the random effect model was used. HR $>1$ for PFS and OS indicated that the anti-VEGF TKI treatment group derived more progression or fatalities. RR $>1$ for ORR and AEs indicated that the anti-VEGF TKI treatment group derived more overall response or more toxicities. The potential presence of publication bias was evaluated visually by inspecting funnel plots and statistically using the Egger's test.

\section{Results}

Included studies. A total of 273 potentially relevant citations were reviewed, and following exclusion of 264 as they were reviews studies, basic researches or case reports, 9 potential RCTs were identified and the full text for each study was screened. Of these, 5 studies were excluded due to incomplete data, phase I pharmacokinetics and tolerability or irrelevant data to compare VEGFR TKI with bevacizumab in mCRC. Finally, 4 randomized trials were eligible for inclusion in the meta-analysis. The study search process is shown in Fig. 1. Four trials with a total of 1,929 intent-to-treat patients included in the meta-analysis were RCTs, and the full text was published in English. Two were double-blind, phase III RCTs $(18,19)$ and 2 were open-label, phase II RCTs $(20,21)$. The main characteristics of all the eligible RCTs are listed in Table I. The trial conducted by Cunningham et al (18) compared cediranib (20 and $30 \mathrm{mg}$ once daily) plus mFOLFOX6 with bevacizumab plus mFOLFOX6, respectively. Only the comparative data between the cediranib 20-mg group and the bevacizumab group were included in the analysis in order to reduce heterogeneity. The trial conducted by Bendell et al (21) compared axitinib/FOLFIRI and axitinib/mFOLFOX6 with FOLFIRI alone and mFOLFOX6 alone, respectively. Therefore, the trial was included in the analysis as 2 independent studies (Bendell-1 2013 and Bendell-2 2013). 


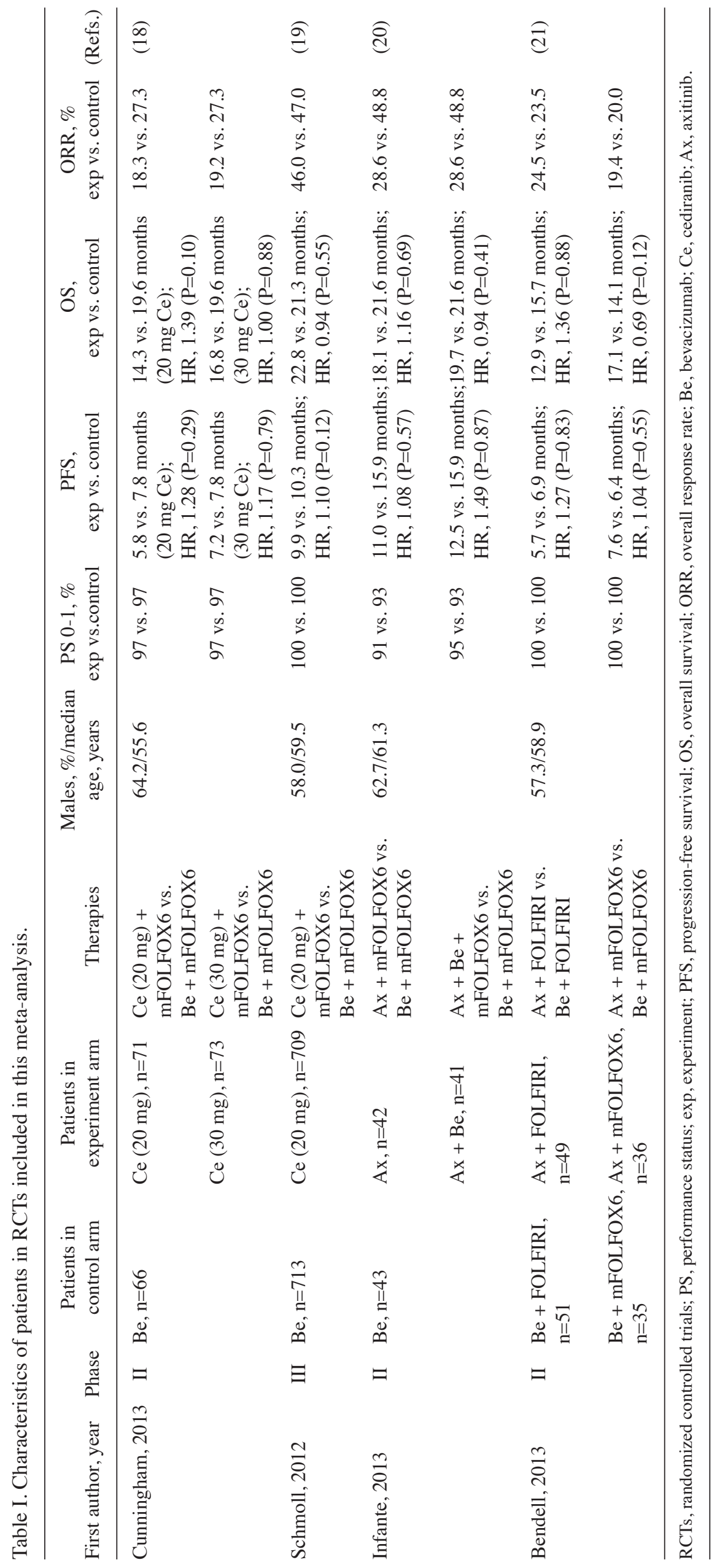




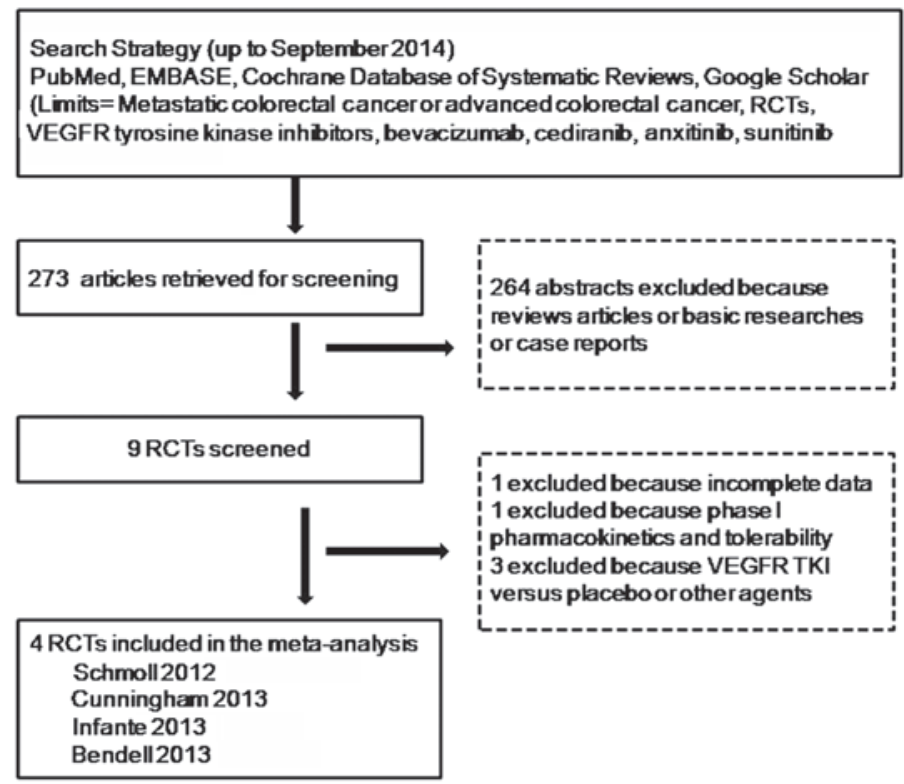

Figure 1. Consort diagram of study selection. RCT, randomized controlled trial; VEGFR TKI, vascular endothelial growth factor receptor tyrosine kinase inhibitors.

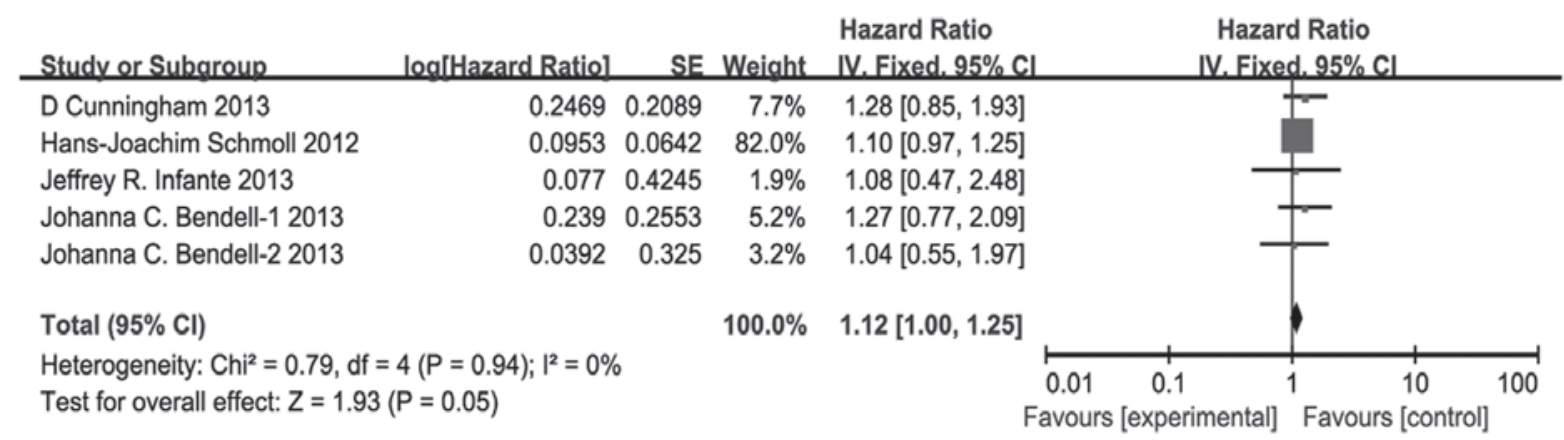

Figure 2. Hazard ratio for progression-free survival with vascular endothelial growth factor receptor tyrosine kinase inhibitors versus bevacizumab in combination with chemotherapy. SE, standard error; CI, confidence interval.

The mean Jadad score was 3.3 for the included studies (Table II). All the trials were randomized, but only 1 described the methods of randomization. Two were double-blind, phase III trials and 2 were open-label, phase II trials; all 4 trials reported their withdrawals and dropouts.

All the studies included in the meta-analysis were approved by the institutional review board or independent ethics committee of each participating center, followed the guiding principles of the Declaration of Helsinki and good clinical practice, and complied with all local laws and regulations. All the patients provided written informed consent prior to enrolment.

Efficacy of VEGFR TKIs plus chemotherapy versus bevacizumab plus chemotherapy in metastatic colorectal cancer

$P F S$. The combination of VEGFR TKIs and chemotherapy resulted in a significant decline in PFS compared with bevacizumab plus chemotherapy (HR, 1.12; 95\% CI, 1.00-1.25; $\mathrm{P}=0.05$ ) (Fig. 2). There was no significant heterogeneity $\left(\mathrm{P}=0.94, \mathrm{I}^{2}=0 \%\right)$, and the pooled HR for PFS was performed using the fixed-effect model.
$O S$. There was no significant difference between the VEGFR TKIs and bevacizumab groups for the pooled HR for OS (HR, 1.01; 95\% CI, 0.88-1.17; $\mathrm{P}=0.87$ ) (Fig. 3). There was no significant heterogeneity $\left(\mathrm{P}=0.20, \mathrm{I}^{2}=33 \%\right)$ and the pooled HR for OS was also performed using the fixed-effect model.

$O R R$. There was no significant difference between the VEGFR TKIs group and bevacizumab group for the pooled RR for ORR (RR, 0.95; 95\% CI, 0.85-1.05; P=0.30) (Fig. 4). There was no significant heterogeneity $\left(\mathrm{P}=0.36, \mathrm{I}^{2}=8 \%\right)$ and the pooled $\mathrm{HR}$ for OS was also performed using the fixed-effect model.

Toxicities of anti-VEGFR TKIs plus chemotherapy versus bevacizumab plus chemotherapy in metastatic colorectal cancer. Reported grade-III/IV adverse reactions in these 4 studies included diarrhea, fatigue, hypertension, neutropenia, peripheral neuropathy, thrombocytopenia, vomiting and abdominal pain. All studies reported diarrhea, fatigue, hypertension, neutropenia, thrombocytopenia and peripheral neuropathy in patients, while 4 reported vomiting and abdominal pain. The comparison of grade-III/IV adverse reactions between the VEGFR TKIs and bevacizumab 
Table II. Jadad score calculation for included studies.

Jadad score calculation parameters
Cunningham et al (18) Schmoll et al (19) Infante et al (20) Bendell et al (21)

Was the study described as randomized

(this includes words such as randomly,

random and randomization)?

Was the method used to generate the sequence of randomization described and appropriate (such as table of random numbers and computer-generated)?

Was the study described as double blind?

Was the method of double blinding described and appropriate (such as identical placebo, active placebo and dummy)?

Was there a description of withdrawals and dropouts?

Total

$\begin{array}{llll}1 & 1 & 1 & 1 \\ 0 & 0 & 1 & 0 \\ 1 & & & \\ 1 & 1 & 0 & 0 \\ 1 & 1 & 0 & 0 \\ 4 & 1 & 1 & 1 \\ & 4 & 3 & 2\end{array}$

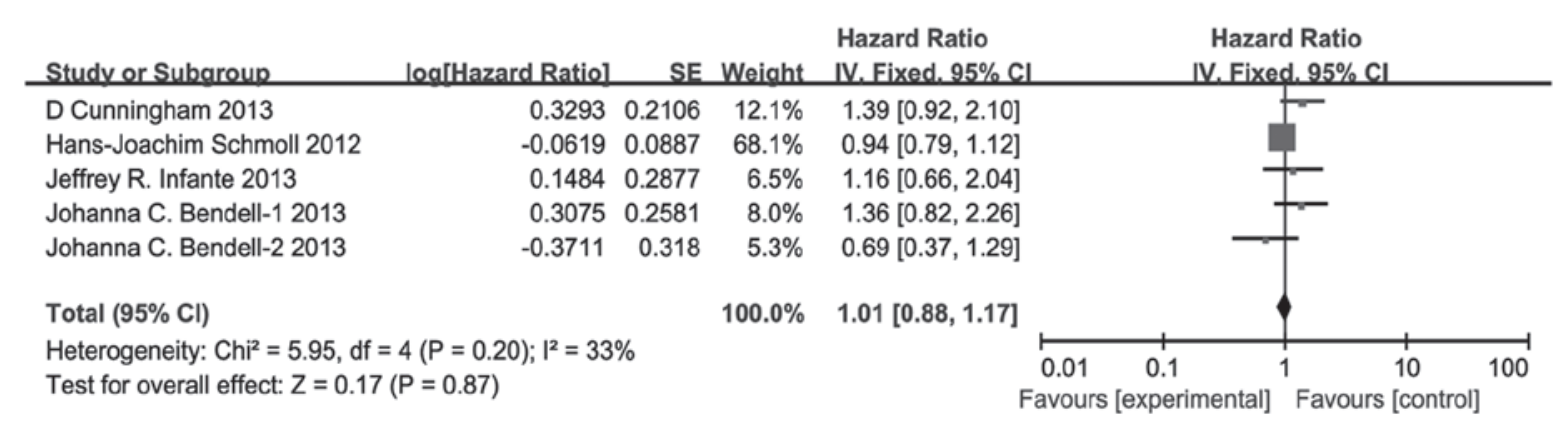

Figure 3. Hazard ratio for overall survival with vascular endothelial growth factor receptor tyrosine kinase inhibitors vs. bevacizumab in combination with chemotherapy. SE, standard error; CI, confidence interval.

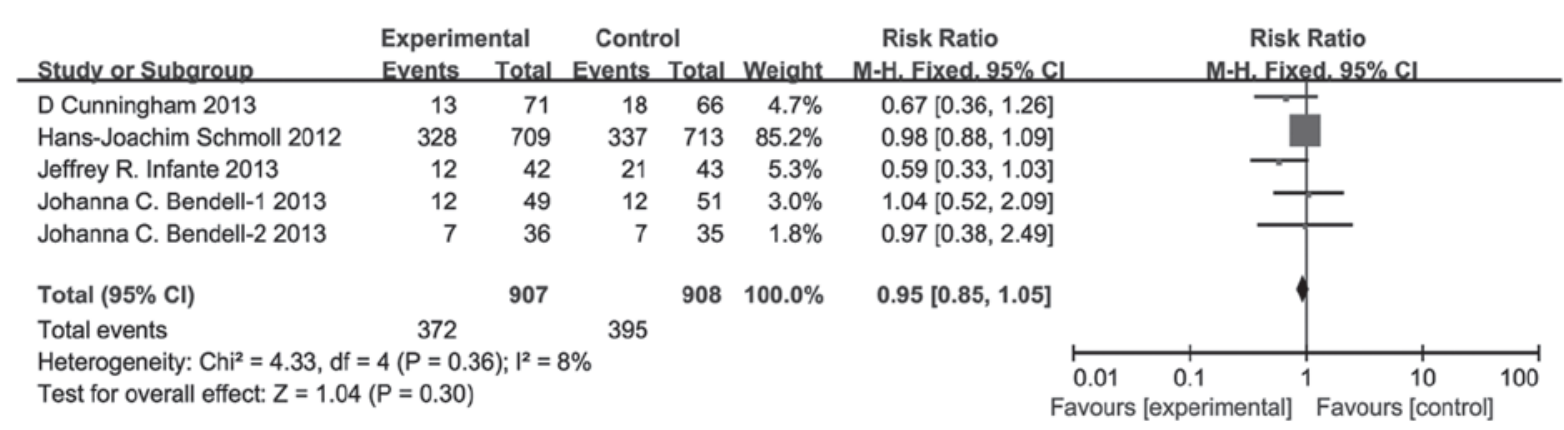

Figure 4. Relative risk for overall response rate with vascular endothelial growth factor receptor tyrosine kinase inhibitors vs. bevacizumab in combination with chemotherapy. SE, standard error; CI, confidence interval.

groups are shown in Fig. 5. The statistically significant differences in pooled estimates suggest a higher incidence of grade-III/IV diarrhea (RR, 2.01; 95\% CI, 1.52-2.66; $\mathrm{P}<0.00001)$, grade-III/IV fatigue ( $\mathrm{RR}, 1.56$; $95 \% \mathrm{CI}, 1.12-2.18$; $\mathrm{P}=0.009)$, grade-III/IV hypertension (RR, 1.67; 95\% CI, 1.15-2.43; $\mathrm{P}=0.007)$, grade-III/IV neutropenia (RR, 1.33; 95\% CI, 1.14-1.54; P=0.0002) and grade-III/IV thrombocytopenia ( $\mathrm{RR}, 2.44 ; 95 \% \mathrm{CI}, 1.48-4.02 ; \mathrm{P}=0.0005$ ) associated with the anti-VEGFR TKI group, particularly for diarrhea and thrombocytopenia. However, the statistically significant differences in pooled estimates suggest a higher incidence of grade-III/IV peripheral neuropathy (RR, 0.72; 95\% CI, 0.52-0.99; $\mathrm{P}=0.05)$ associated with the bevacizumab group. No statistically significant differences were noted in the incidence of grade-III/IV vomiting (RR, 0.96; 95\% CI, 0.41-2.25; $\mathrm{P}=0.92)$ and grade-III/IV abdominal pain (RR, 1.64; 95\% CI, 0.62-4.34; P=0.32).

\section{Discussion}

Agents targeting the angiogenic pathway have been the cornerstone of mCRC treatment in recent years. The survival 


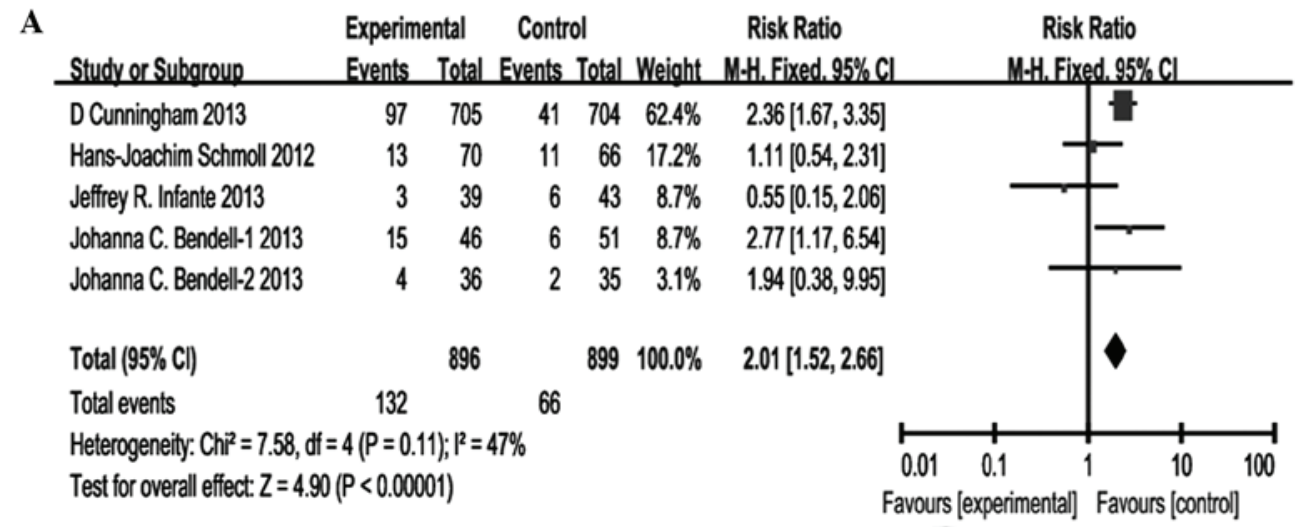

B

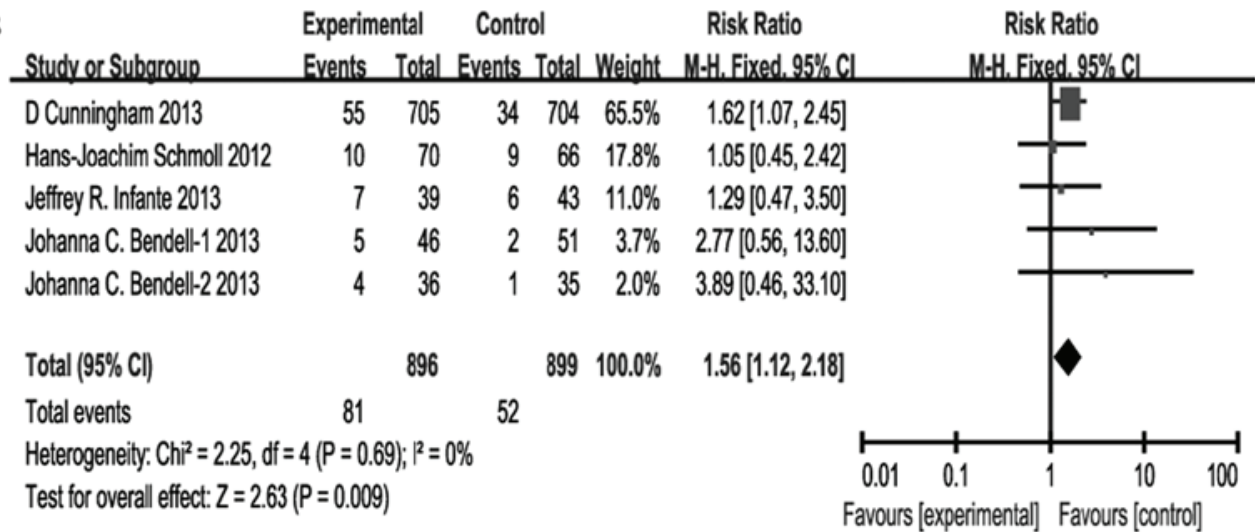

C

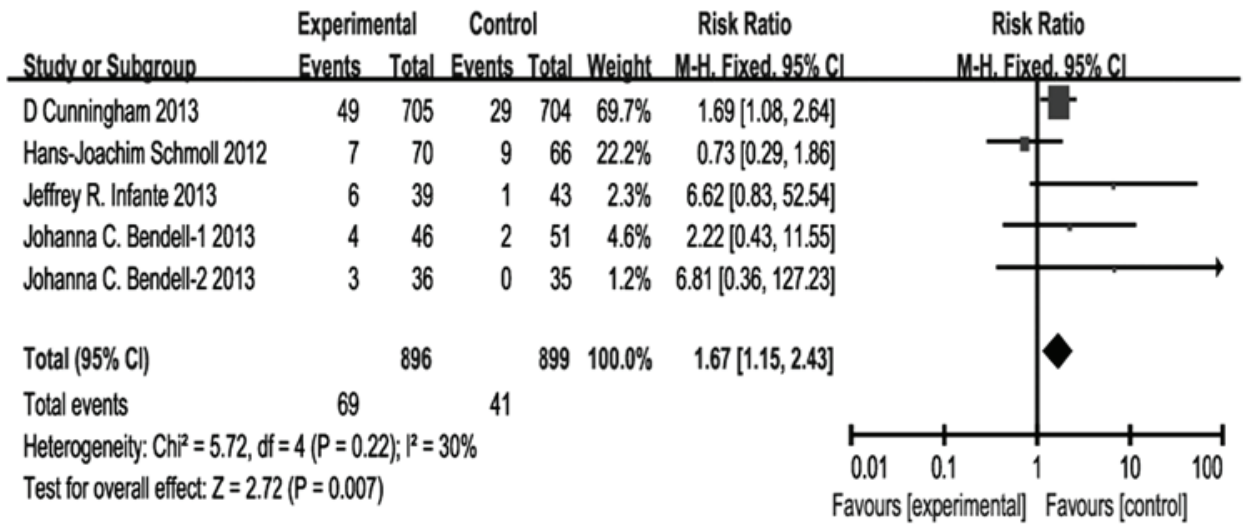

D

\begin{tabular}{|c|c|c|c|c|c|c|c|}
\hline \multirow[b]{2}{*}{ Study or Suboroup } & \multicolumn{2}{|c|}{ Experimental } & Control & \multirow{2}{*}{\multicolumn{2}{|c|}{$\begin{array}{c}\text { Risk Ratio } \\
\text { tal Weight_M.H. Fixed, } 95 \% \text { C }\end{array}$}} & \multirow{2}{*}{\multicolumn{2}{|c|}{$\begin{array}{l}\text { Risk Ratio } \\
\text { M-H. Fixed, } 95 \% \mathrm{CL}\end{array}$}} \\
\hline & & Iotal & Events _ I & & & & \\
\hline D Cunningham 2013 & 224 & 705 & 166 & $704 \quad 76.1 \%$ & $1.35[1.14,1.60]$ & & \\
\hline Hans-Joachim Schmoll 2012 & 26 & 70 & 19 & $66 \quad 9.0 \%$ & $1.29[0.79,2.10]$ & & \\
\hline Jeffrey R. Infante 2013 & 13 & 39 & 13 & $43 \quad 5.7 \%$ & $1.10[0.58,2.08]$ & & \\
\hline Johanna C. Bendell-1 2013 & 14 & 46 & 17 & $51 \quad 7.4 \%$ & $0.91[0.51,1.64]$ & & \\
\hline Johanna C. Bendell-2 2013 & 12 & 36 & 4 & $35 \quad 1.9 \%$ & $2.92[1.04,8.18]$ & & \\
\hline Total (95\% Cl) & & 896 & & $899 \quad 100.0 \%$ & $1.33[1.14,1.54]$ & & 4 \\
\hline Total events & 289 & & 219 & & & & \\
\hline $\begin{array}{l}\text { Heterogeneity: Chi }=4.18 \text {, df } \\
\text { Test for overall effect: } Z=3.70\end{array}$ & $\begin{array}{l}4 P=0.38 \\
(P=0.0002\end{array}$ & & & & & $\begin{array}{ll}0.01 & 0.1 \\
\text { Favours [experimental] }\end{array}$ & al 110100 \\
\hline
\end{tabular}

Figure 5. Relative risks of grade-III/IV adverse reactions between vascular endothelial growth factor receptor tyrosine kinase inhibitors versus bevacizumab in combination with chemotherapy. (A) Grade-III/IV diarrhea; (B) grade-III/IV fatigue; (C) grade-III/IV hypertension and (D) grade-III/IV neutropenia. CI, confidence interval. 


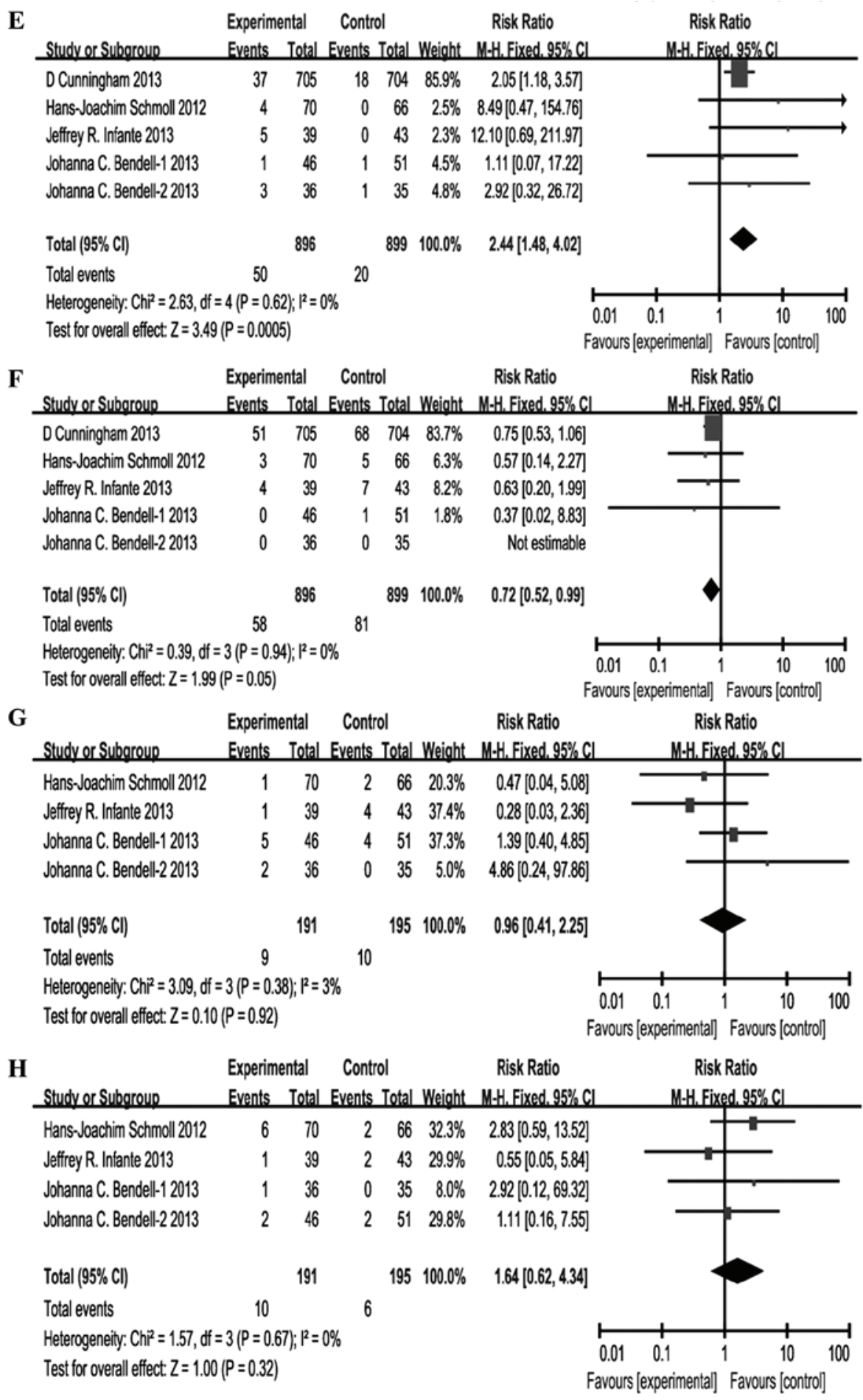

Figure 5. Continued. (E) Grade-III/IV thrombocytopenia; (F) grade-III/IV peripheral neuropathy; (G) grade-III/IV vomiting; and (H) grade-III/IV abdominal pain. CI, confidence interval.

benefit of adding bavacizumab to chemotherapy has been demonstrated in a number of randomized clinical studies, and consequently, the combination of bevacizumab with FOLFOX is the preferred front-line regimen amongst US clinicians (22). Since then, additional randomized studies have shown the antitumor activity of other VEGF-targeted therapies (23). Recently, several RCTs comparing the efficacy and toxicity of VEGFR TKIs against bevacizumab have been reported, but the majority have shown inadequate results. In the HORIZON III trial, cediranib in combination with mFOLFOX6 showed comparable clinical activity to bevacizumab plus mFOLFOX6 in first-line mCRC but failed to meet the predefined boundary for cediranib PFS non-inferiority. Similarly, in the HORIZON I trial, cediranib had antitumor activity in patients with previously treated mCRC, with no statistically significant differences observed in PFS, OS and ORR comparisons with bevacizumab. However, Infante et al (20) demonstrated that neither the addition of continuous axitinib nor the axitinib/bevacizumab combination to FOLFOX-6 improved ORR, PFS or OS compared 
with bevacizumab as first-line treatment of mCRC. In another study, Bendell et al (21) showed that axitinib did not improve outcomes when added to second-line chemotherapy compared with bevacizumab for mCRC. The present meta-analysis focused on the RCTs comparing the efficacy and toxicity of VEGFR TKIs against bevacizumab to identify the potentially significant benefit with the combined treatment regimens.

The results confirmed that VEGFR TKIs plus chemotherapy significantly resulted in a modest but significantly shorter PFS (HR, 1.12; $\mathrm{P}=0.05)$ compared with bevacizumab plus chemotherapy. However, there were no statistically significant differences in OS (HR, 1.10; 95\% CI, 0.88-1.17; $\mathrm{P}=0.87$ ) and ORR (RR, 0.95; 95\% CI, 0.85-1.05; $\mathrm{P}=0.30$ ) between the treatment arms. As for the safety profile, the VEGFR TKIs group showed a less favorable AE profile compared with bevacizumab, with higher rates of grade-III/IV diarrhea, fatigue, hypertension, neutropenia and thrombocytopenia, whereas a higher incidence of peripheral neuropathy was associated with the bevacizumab group.

The efficacy outcomes of bevacizumab in the included studies, in terms of PFS and OS, were consistent with the earlier studies of this agent in combination with chemotherapy in firstor second-line mCRC. Median PFS in the first-line setting [10.3 months, Schmoll et al (19); 15.9 months, Infante et al (20)] was comparable to that observed with bevacizumab plus FOLFOX4/CAPOX [9.4 months; Van Cutsem et al (24)], PFS in the second-line setting [7.8 months, Cunningham et al (18); 6.9 months and 6.4 months, Bendell et al (21)] was also comparable to that observed with vatalanib (PTK787/ZK) plus mFOLFOX6 [5.6 months; Van Cutsem et al (24)]. OS data were similar to the earlier studies.

Cediranib and axitinib are highly potent VEGFR TKIs with in vivo activity against all three VEGF receptors and may result in more complete blockade of VEGF to additive antitumor activity compared with bevacizumab, however, it was confirmed that neither the addition of cediranib nor axitinib combination to chemotherapy improved ORR, PFS or OS compared with bevacizumab as first- or second-line treatment of mCRC. Furthermore, AEs leading to discontinuation, dose reduction or dose interruption were reported more frequently for VEGFR TKIs treatment arms, which may lead to fewer cycles and lower dose intensity of chemotherapy. The meta-analysis showed that VEGFR TKIs plus chemotherapy significantly increased the risk of progression by $12 \%$ compared with bevacizumab (HR 1.12; $\mathrm{P}=0.05$ ). More tolerability issues and lower dose intensity of chemotherapy may also be important factors to consider.

In the present meta-analysis, patients who received TKIs had higher incidences of grade-III/IV diarrhea, fatigue, hypertension, neutropenia and thrombocytopenia, which were consistent with the safety profiles of other VEGFR TKIs (25-27). The pooled analysis showed that the rate of diarrhea and thrombocytopenia were more than twice as high with the addition of VEGFR TKI to chemotherapy, which may be associated with the antitumor activities of 5-FU/LV. Hypertension may be a class and common effect of angiogenesis inhibitors although the mechanisms of hypertension are unclear. There was a higher incidence of peripheral neuropathy in the bevacizumab group and it may account for more cycles and a higher dose intensity of chemotherapy compared with VEGFR TKI. In general, it appears that VEGFR TKIs plus chemotherapy were not as well-tolerated as bevacizumab-based regimens and may result in early discontinuations and decreased dose intensity of all the agents.

Although the previous studies conducted with additional VEGFR TKIs to chemotherapy have been unsuccessful to date $(24,25,28,29)$, the potential use of oral VEGFR TKIs continues to be investigated. Recently, it was demonstrated that the oral VEGFR TKI regorafenib improved OS and PFS compared with the placebo in patients with advanced CRC who had received previous treatment with oxaliplatin and irinotecan-based chemotherapy (30), which showed promise for treatment of mCRC. Further investigation with this class of agent is warranted in pursuit of effective clinical treatment. Markers that are predictive for response to VEGFR therapy have not yet been identified but are undergoing investigation, which may reveal a benefit in select patient populations in the future (31).

In conclusion, based on the results of the present meta-analysis, VEGFR TKIs plus chemotherapy significantly resulted in a modest but significantly shorter PFS compared with bevacizumab plus chemotherapy but not in OS and ORR. VEGFR TKI treatment showed a less favorable AE profile compared with bevacizumab, with higher rates of grade-III/IV diarrhea, fatigue, hypertension, neutropenia and thrombocytopenia, whereas a higher incidence of peripheral neuropathy was associated with bevacizumab.

\section{Acknowledgements}

The authors wish to acknowledge Professor Xuejun Lao and, in particular, Dr Fang Lu, Dr Yongliang Huang and Dr Junjie Liang for their support in the development of the study, and Dr Yiling Yang for the revision of the manuscript.

\section{References}

1. Siegel R, Naishadham D and Jemal A: Cancer statistics, 2012. CA Cancer J Clin 62: 10-29, 2012.

2. Engstrom PF, Arnoletti JP, Benson AB III, Chen YJ, Choti MA, Cooper HS, Covey A, Dilawari RA, Early DS, Enzinger PC, et al; National Comprehensive Cancer Network: NCCN Clinical Practice Guidelines in Oncology: Colon cancer. J Natl Compr Canc Netw 7: 778-831, 2009.

3. Tournigand C, André T, Achille E, Lledo G, Flesh M, Mery-Mignard D, Quinaux E, Couteau C, Buyse M, Ganem G, et al: FOLFIRI followed by FOLFOX6 or the reverse sequence in advanced colorectal cancer: A randomized GERCOR study. J Clin Oncol 22: 229-237, 2004.

4. Ferrara N: Vascular endothelial growth factor as a target for anticancer therapy. Oncologist 9 (Suppl 1): 2-10, 2004.

5. Giantonio BJ, Catalano PJ, Meropol NJ, O'Dwyer PJ, Mitchell EP, Alberts SR, Schwartz MA and Benson AB III; Eastern Cooperative Oncology Group Study E3200: Bevacizumab in combination with oxaliplatin, fluorouracil, and leucovorin (FOLFOX4) for previously treated metastatic colorectal cancer: Results from the Eastern Cooperative Oncology Group Study E3200. J Clin Oncol 25: 1539-1544, 2007.

6. Wedge SR, Kendrew J, Hennequin LF, Valentine PJ, Barry ST, Brave SR, Smith NR, James NH, Dukes M, Curwen JO, et al: AZD2171: A highly potent, orally bioavailable, vascular endothelial growth factor receptor-2 tyrosine kinase inhibitor for the treatment of cancer. Cancer Res 65: 4389-4400, 2005.

7. Drevs J, Medinger M, Mross K, et al: Phase I clinical evaluation of AZD2171, a highly potent VEGF receptor tyrosine kinase inhibitor, in patients with advanced tumors. J Clin Oncol 23: 192s, 2005. 
8. Hoff PM,Hochhaus A, Pestalozzi BC, Tebbutt NC, Li J, Kim TW, Koynov KD, Kurteva G, Pintér T, Cheng Y, et al: Cediranib plus FOLFOX/CAPOX versus placebo plus FOLFOX/CAPOX in patients with previously untreated metastatic colorectal cancer: A randomized, double-blind, phase III study (HORIZON II). J Clin Oncol 30: 3596-3603, 2012.

9. Hu-Lowe DD, Zou HY, Grazzini ML, Hallin ME, Wickman GR, Amundson K, Chen JH, Rewolinski DA, Yamazaki S, Wu EY, et al: Nonclinical antiangiogenesis and antitumor activities of axitinib (AG-013736), an oral, potent, and selective inhibitor of vascular endothelial growth factor receptor tyrosine kinases 1, 2, 3. Clin Cancer Res 14: 7272-7283, 2008.

10. Cohen EE, Rosen LS, Vokes EE, Kies MS, Forastiere AA, Worden FP, Kane MA, Sherman E, Kim S, Bycott P, et al: Axitinib is an active treatment for all histologic subtypes of advanced thyroid cancer: Results from a phase II study. J Clin Oncol 26: 4708-4713, 2008.

11. Fruehauf J, Lutzky J, McDermott D, Brown CK, Meric JB, Rosbrook B, Shalinsky DR, Liau KF, Niethammer AG, Kim S, et al: Multicenter, phase II study of axitinib, a selective second-generation inhibitor of vascular endothelial growth factor receptors 1,2 , and 3 , in patients with metastatic melanoma. Clin Cancer Res 17: 7462-7469, 2011.

12. Rini BI, Wilding G, Hudes G, Stadler WM, Kim S, Tarazi J, Rosbrook B, Trask PC, Wood L and Dutcher JP: Phase II study of axitinib in sorafenib-refractory metastatic renal cell carcinoma. J Clin Oncol 27: 4462-4468, 2009.

13. Rixe O, Bukowski RM, Michaelson MD, Wilding G, Hudes GR, Bolte O, Motzer RJ, Bycott P, Liau KF, Freddo J, et al: Axitinib treatment in patients with cy tokine-refractory metastatic renal-cell cancer: A phase II study. Lancet Oncol 8: 975-984, 2007.

14. Schiller JH, Larson T, Ou SH, Limentani S, Sandler A, Vokes E, Kim S, Liau K, Bycott P, Olszanski AJ, et al: Efficacy and safety of axitinib in patients with advanced non-small-cell lung cancer: Results from a phase II study. J Clin Oncol 27: 3836-3841, 2009.

15. Jadad AR, Moore RA, Carroll D, Jenkinson C, Reynolds DJ, Gavaghan DJ and McQuay HJ: Assessing the quality of reports of randomized clinical trials: Is blinding necessary? Control Clin Trials 17: 1-12, 1996.

16. Kjaergard LL, Villumsen J and Gluud C: Reported methodologic quality and discrepancies between large and small randomized trials in meta-analyses. Ann Intern Med 135: 982-989, 2001.

17. Moher D, Pham B, Jones A, Cook DJ, Jadad AR, Moher M, Tugwell P and Klassen TP: Does quality of reports of randomised trials affect estimates of intervention efficacy reported in meta-analyses? Lancet 352: 609-613, 1998.

18. Cunningham D, Wong RP, D'Haens G, Douillard JY, Robertson J, Stone AM and Van Cutsem E; HORIZON I study group: Cediranib with mFOLFOX6 vs. bevacizumab with mFOLFOX6 in previously treated metastatic colorectal cancer. Br J Cancer 108: 493-502, 2013.

19. Schmoll HJ, Cunningham D, Sobrero A, Karapetis CS, Rougier P, Koski SL, Kocakova I, Bondarenko I, Bodoky G, Mainwaring P, et al: Cediranib with mFOLFOX6 versus bevacizumab with mFOLFOX6 as first-line treatment for patients with advanced colorectal cancer: A double-blind, randomized phase III study (HORIZON III). J Clin Oncol 30: 3588-3595, 2012.

20. Infante JR, Reid TR, Cohn AL, Edenfield WJ, Cescon TP, Hamm JT, Malik IA, Rado TA, McGee PJ, Richards DA, et al: Axitinib and/or bevacizumab with modified FOLFOX-6 as first-line therapy for metastatic colorectal cancer: A randomized phase 2 study. Cancer 119: 2555-2563, 2013.
21. Bendell JC, Tournigand C, Swieboda-Sadlej A, Barone C, Wainberg ZA, Kim JG, Pericay C, Pastorelli D, Tarazi J, Rosbrook B, et al: Axitinib or bevacizumab plus FOLFIRI or modified FOLFOX-6 after failure of first-line therapy for metastatic colorectal cancer: A randomized phase II study. Clin Colorectal Cancer 12: 239-247, 2013.

22. Colucci G, Gebbia V, Paoletti G, Giuliani F, Caruso M, Gebbia N, Cartenì G, Agostara B, Pezzella G, Manzione L, et al; Gruppo Oncologico Dell'Italia Meridionale: Phase III randomized trial of FOLFIRI versus FOLFOX4 in the treatment of advanced colorectal cancer: A multicenter study of the Gruppo Oncologico Dell'Italia Meridionale. J Clin Oncol 23: 4866-4875, 2005.

23. Saltz LB, Clarke S, Díaz-Rubio E, Scheithauer W, Figer A, Wong R, Koski S, Lichinitser M, Yang TS, Rivera F, et al: Bevacizumab in combination with oxaliplatin-based chemotherapy as first-line therapy in metastatic colorectal cancer: A randomized phase III study. J Clin Oncol 26: 2013-2019, 2008.

24. Van Cutsem E, Bajetta E, Valle J, Köhne CH, Hecht JR, Moore M, Germond C, Berg W, Chen BL, Jalava T, et al: Randomized, placebo-controlled, phase III study of oxaliplatin, fluorouracil, and leucovorin with or without PTK787/ZK 222584 in patients with previously treated metastatic colorectal adenocarcinoma. J Clin Oncol 29: 2004-2010, 2011.

25. Hecht JR, Trarbach T, Hainsworth JD, Major P, Jäger E, Wolff RA, Lloyd-Salvant K, Bodoky G, Pendergrass K, Berg W, et al: Randomized, placebo-controlled, phase III study of first-line oxaliplatin-based chemotherapy plus PTK787/ZK 222584, an oral vascular endothelial growth factor receptor inhibitor, in patients with metastatic colorectal adenocarcinoma. J Clin Oncol 29: 1997-2003, 2011.

26. Llovet JM, Ricci S, Mazzaferro V, Hilgard P, Gane E, Blanc JF, de Oliveira AC, Santoro A, Raoul JL, Forner A, et al; SHARP Investigators Study Group: Sorafenib in advanced hepatocellular carcinoma. N Engl J Med 359: 378-390, 2008.

27. Schutz FA, Je Y and Choueiri TK: Hematologic toxicities in cancer patients treated with the multi-tyrosine kinase sorafenib: A meta-analysis of clinical trials. Crit Rev Oncol Hematol 80: 291-300, 2011.

28. Carrato A, Swieboda-Sadlej A, Staszewska-Skurczynska M, Lim R, Roman L, Shparyk Y, Bondarenko I, Jonker DJ, Sun Y, De la Cruz JA, et al: Fluorouracil, leucovorin, and irinotecan plus either sunitinib or placebo in metastatic colorectal cancer: A randomized, phase III trial. J Clin Oncol 31: 1341-1347, 2013.

29. Tabernero J, Garcia-Carbonero R, Cassidy J, Sobrero A, Van Cutsem E, Köhne CH, Tejpar S, Gladkov O, Davidenko I, Salazar R, et al: Sorafenib in combination with oxaliplatin, leucovorin, and fluorouracil (modified FOLFOX6) as first-line treatment of metastatic colorectal cancer: The RESPECT trial. Clin Cancer Res 19: 2541-2550, 2013.

30. Grothey A, Van Cutsem E, Sobrero A, Siena S, Falcone A, Ychou M, Humblet Y, Bouché O, Mineur L, Barone C, et al; CORRECT Study Group: Regorafenib monotherapy for previously treated metastatic colorectal cancer (CORRECT): An international, multicentre, randomised, placebo-controlled, phase 3 trial. Lancet 381: 303-312, 2013

31. Schmoll H, Hoff PM, Robertson JD, et al: Association of baseline CEA, VEGF, and soluble VEGF receptor-2 with treatment outcomes in two randomized phase III trials of cediranib in metastatic colorectal cancer (mCRC). J Clin Oncol 29 (Suppl): 3590, 2011. 